\title{
Windows On Language Evolution: What Are They And Wherein Lies Their Virtue?*
}

\author{
Rudolf P Botha \\ Department of General Linguistics, Stellenbosch University, 7600 \\ Stellenbosch, South Africa. E-mail: rpb@sun.ac.za
}

\section{Introduction}

Accounts of the evolution of human language must, by their very nature, express claims of a historical sort, including claims about why, when, where or how human language emerged and/or developed in some distant past. What is more, it is of the essence of these claims that they are put forward in the absence of direct evidence - contained in natural or man-made records - about the events and factors that may or may not have been involved in the evolution of language. In modern work on language evolution, however, scholars have come up with various means of ameliorating this problem of evidential paucity. One of these is an approach that proceeds from the assumption that language evolution can be studied by examining other phenomena about which there is direct evidence. These other phenomena are taken to offer windows on the evolution of language. Thus it has been contended that features of language evolution can be "seen" by "looking at" them through windows offered by prehistoric stone tools, fossilized (fragments of) ancestral skulls, bird song, "language" genes, motherese, pidgin languages or homesigns created by deaf children of non-signing parents to mention just a few.

"This article evolved from a paper presented at a seminar with the theme "Windows on Language Genesis" that was held on 7 - 8 November 2003 at the Netherlands Institute for Advanced Study. 
In a number of respects, the Windows Approach to the study of language evolution, however, is understood rather less than well. This article, accordingly, addresses the following general questions:

(a) What is a window on language evolution?

(b) What are the features that make individual windows on language evolution more or less virtuous?

(c) What are the virtues of the Windows Approach to the study of language evolution?

These are not the only questions that arise in connection with the Windows Approach. An important further question is: What is involved in constructing a window on language evolution? This question, however, falls outside the limited scope of the present paper and is the topic of a following one (= Botha, 2004b).

\section{2. "Language evolution"}

As for question (1)(a) - "What is a window on language evolution?" - I would like to begin addressing it by offering a clarification of the compound concept of "language evolution". Its components - "language" and "evolution" - have been used in the literature in some ways that are more restrictive (or constrained) and other ways that are less restrictive (or constrained). Used restrictively, the concept of "language" includes -

(2) (a) a human capacity, referred to by such expressions as "the human language capacity" and "the human language faculty"; and

(b) the system(s) referred to by such expressions as "the first human language", "ancestral language", "the first form of human language". ${ }^{1}$

In the restrictive senses of (2), the concept of "language" does not include what has been referred to as "speech" or "mechanisms involved in speaking or listening".

A sizeable volume of recent work on language evolution, however, adopts a less restrictive concept of "language" in terms of which "language" appears in many guises, including those referred to as - 
"(group) behaviour", "skill", "activity", "process", "meta-task", "sort of (social) contract", "something formed by itself", "multimodal enhancement system", "(form of) communication" and so on. ${ }^{2}$

Accounts of language evolution that have used the concept of "language" in unconstrained senses such as these have been shown to be generally opaque: it is unclear what the entity or entities are whose evolution is at issue. And this has resulted in discussions of issues in language evolution that are internally disconnected and debates that are strikingly inconclusive. People are disagreeing about what the evolution of "language" involves but are often not talking about the evolution of the same thing. ${ }^{3}$ Work that adopts a restrictive concept of "language" that includes (2)(a) and (b), but only(2)(a) and (b), does not deny that there are distinct linguistic entities that can be denoted by the expressions "speech", "behaviour", "skill" and so on. Nor does it deny that such entities may have evolutionary histories that can be properly investigated in their own right. What such work denies is that the evolution of language can be insightfully studied with the aid of a concept within which the entities referred to in (2) and (3) are collapsed into one entity with a single evolutionary history.

Which brings me to the concept of "evolution", the other component of the concept of "language evolution". In modern work that uses the concept of "evolution" in a restrictive way, evolution represents a process comprising various phases, central to which are the two referred to as -

\footnotetext{
(a) "origin, emergence or first appearance (of language in the human species)"; and

(b) "subsequent development (of language in the human species)". ${ }^{4}$
}

Used in this restrictive sense, the concept of " evolution" applies to the phenomenon that has also been referred to as "language phylogeny/phylogenesis". The phase referred to as "subsequent development" is sometimes assumed to be made up of a number of subphases as well - an assumption which it is unnecessary to flesh out here.

In the sense of "language phylogeny", language evolution differs at first blush from other processes by which linguistic objects are created, acquired or changed. These processes include those referred to as - 

(a) "the creation of pidgins",
(b) "the emergence of creoles",
(c) "the creation of homesigns by deaf children with hearing parents",
(d) "the emergence of full-blown sign languages",
(e) "the formation of individual (full) languages and language families through processes of diachronic change and diversification", and / or
(f) "the acquisiton of spoken and signed languages by children and adults".

The expression "language genesis" is used in the literature to refer to both the phylogenetic processes (4)(a) and (b) and the genetic processes (5)(a) - (f). ${ }^{5}$ There is no harm in this terminological practice as long as it is kept in mind that to establish the ways in which the various genetic processes are similar or different requires empirical work of a substantive sort, a point to which I will be returning below. In the present paper, the expression "language evolution" is taken in the restrictive sense of (4).

\section{3. "Window"}

This brings us to the concept of a "window (on language evolution)". In general terms, a phenomenon $\mathrm{X}$ is considered (to offer) a window on a distinct phenomenon $\mathrm{Y}$ if by "looking at" $\mathrm{X}$ it is possible to "see" something of Y. A window on language evolution, accordingly, is a phenomenon that has properties believed to offer a "view" on properties of some aspect or aspects of language evolution. To put the matter in this way is, of course, to give a metaphorical characterization of what a window on language evolution is.

So what would a window on language evolution be in non-metaphorical terms? In nonmetaphorical terms, a window is in essence a device for making inferences about language evolution. To - metaphorically - "see" a property of some aspect of language evolution by metaphorically - "looking at" a property of some other phenomenon is - nonmetaphorically to infer that first property from data about this second property. As inferential devices, windows on language evolution have a structure that may be roughly portrayed as follows: 
(6)

\begin{tabular}{|l|}
\hline Data about some proper- \\
ty/properties of a \\
phenomenon that itself is \\
distinct from language \\
evolution (in the \\
restrictive sense)
\end{tabular}

(a)

\begin{tabular}{|c|c|}
\hline$\frac{\text { Inferential }}{\operatorname{step}(\mathrm{s})}$ & $\begin{array}{l}\text { Conclusions about some } \\
\text { property/properties of an } \\
\text { aspect of language } \\
\text { evolution (in the } \\
\text { restrictive sense) }\end{array}$ \\
\hline
\end{tabular}

(b) (c)

The use of the expression "window on language evolution" needs to be clarified further in a number of general ways.

First, the expression "window on language evolution" has a more formal and a less formal use. More formally, it refers to devices with the structure portrayed in (6), i.e., devices made up of (minimally) three components - those indicated by (a), (b) and (c). Less formally, by contrast, the expression "window on language evolution" has been used to refer to only the phenomenon or phenomena identified in box (6)(a). That is, in this second use, the expression refers to what may be called more accurately the "window phenomenon" or "window phenomena". The phenomena themselves - the fossil skulls, the pidgins, motherese and so on - are portrayed, in this second use, as windows on language evolution. Where confusion is unlikely to arise, I will use the expression "window on language evolution" in the less formal sense as well.

Second, a distinction has to be made between the expression "window on language evolution" and the concept of "window of language evolution". The concept applies to any instance of an inference where a conclusion about an aspect of language evolution is drawn on the basis of data about a phenomenon that is distinct from language evolution. The latter data have conventionally been said to offer "indirect evidence" for claims about the former aspects of language evolution. ${ }^{6}$ Thus, to instantiate the concept of "window on language evolution", an inferential device of the kind under consideration does not need to have been called a "window on language evolution" in so many words.

We are now in a position to consider question (1)(b): "What are the features that make individual windows on language evolution more or less virtuous?" Though incomplete, the portrayal offered as (6) of the structure of a window on language evolution makes it possible to identify three of the basic features that determine how good such a window is as an 
inferential device. These are features of the inferences which a window allows to be drawn "window inferences", for short - namely those of groundedness, warrantedness and pertinence. In what follows, we will be considering these three "good-making" features of windows on language evolution in some detail.

\section{Groundedness}

As a feature of a window inference, groundedness reflects something about the starting point of the inference, specifically about the window phenomenon identified in box (a) in schema (6). A window inference can be considered grounded to the extent that it is based on a window phenomenon whose properties are well-understood. This point can be illustrated with reference to the phenomenon that Ray Jackendoff has referred to as "degraded" forms of language. In two recent publications, Jackendoff $(1999$, p. 273; 2002, p. 238) has made the claim that the emergence of modern language can be broken down into nine partially ordered steps or stages. ${ }^{7}$ In support of this claim, he furnishes evidence from what he considers to be a new source - so-called "language fossils". Language fossils are characterized by him (1999, p. 272) as traces of ancestral language that can be found in what he refers to as "degraded" forms of language. For example, such "degraded" forms of language as the Basic Variety (BV), homesigns invented by deaf children of non-signing parents, pidgin languages and the language of agrammatic aphasics exhibit, in Jackendoff's (1999, pp. 275-276) view, traces of the stage in the emergence of language referred to as "protolanguage". As examples of such traces, Jackendoff (1999, p. 275) cites principles that use the linear order of concatenated symbols for expressing semantic relations. The so-called "fossil" principles of Agent First which says that Agent is expressed in the subject position - and Focus Last - which says that the informationally focal elements appear last in a string - both represent such traces, in his view. The occurrence of these principles in "degraded" forms of language is taken by Jackendoff as evidence for the conclusion that in the emergence of language there was a stage, namely protolanguage, that was likewise characterized by the presence of these principles.

The question, of course, is: How well-understood is the phenomenon of "degraded" (forms of) language from which Jackendoff draws his conclusions about stages in the emergence of language? In this regard, he does not address the question stated in (7):

What is it that makes a form of language "degraded"? 
But if there is no clear answer to question (7), then questions (8)(a) - (c) cannot be given nonarbitrary answers.

(a) How is it to be determined that the form of language $\mathrm{X}$ (or $\mathrm{Y}$ or $\mathrm{Z}$ ) does or does not represent a "degraded" form of language?

(b) How is it to be determined that the linguistic phenomenon P (or Q or R) has the property of "degradedness"?

(c) Must every property associated with every form of linguistic restrictedness be taken as a manifestation of "degradedness"?

If questions such as (7) and (8)(a) - (c) cannot be answered in a clear and non-arbitrary way, then "degraded" language represents a phenomenon that is poorly demarcated and, therefore, not well-understood. This leaves an unwelcome possibility wide open: the possibility of simply stipulating that a particular form of language is a "degraded" form of language. And so it is possible, in turn, to stipulate that a particular property is a manifestation of degradedness and that another one is not. But if it is possible to stipulate what is and what is not a "degraded" form of language, it is possible likewise to stipulate what is and what is not a "language fossil". And this makes it possible to stipulate further what is and what is not a stage in the emergence of language. But, of course, stipulating that some aspect of language genesis has or lacks a particular property is not the same as inferring that it has or lacks this property (Botha, 2003, p.203).

The core of the problem is that Jackendoff does not anywhere offer a characterization of what "degradedness" involves. What he (1999, 275-276) does do is to offer an ad hoc list of examples of "degraded" forms of language. His list seems to have the following five as definite members: homesigns invented by deaf children of non-signing parents, pidgin languages, the language of agrammatic aphasics, BV, and the language used by young children. ${ }^{8}$ But he leaves unclear what it is that makes, for instance, both the language used "early" by children and the language of agrammatic aphasics "degraded". 9

In short: the inferences made by Jackendoff on the basis of data about "degraded" forms of language about the emergence of language are not properly grounded. Specifically, these inferences are not underpinned by an insightful theory of what a "degraded" form of language is. To be insightful, such a theory would have to be restrictive in at least the following sense: 
it would have to give a basis on which to discriminate in a non-arbitrary way between genuine "degraded" forms of language and their deceptive look-alikes: forms of language that may seem "degraded"on the face of it but that are not "degraded"in fact. Of course, though, the problems with demarcating "degraded" language as a unitary phenomenon do not carry over automatically to the demarcation of the individual phenomena which Jackendoff has taken to be instances of "degraded" forms of language. That is, from the fact that "degraded" language is poorly understood and demarcated as a unitary phenomenon it by no means follows as a matter of logical necessity that pidgins, homesigns, the Basic Variety and so on are poorly understood and demarcated individual phenomena.

The conclusion that the inferences drawn about the emergence of language from data about properties of "degraded" forms of language are not properly grounded, signifies two general things. On the negative side: this conclusion indicates that the "degraded" language window on language evolution is not sufficiently well-developed. On the positive side: this same conclusion highlights one of the virtues of the Windows Approach to the study of language evolution. This approach, clearly, is heuristically virtuous in that it generates interesting questions about window phenomena, questions such as (7) and (8)(a) - (c). These questions form a strong stimulus for in-depth investigation into the nature and properties of so-called "degraded" forms of language. Such work should attempt, minimally, to construct a theory that gives a restrictive characterization of what a "degraded" form of language is. Should these attempts fail, there would be good grounds for concluding that "degraded" forms of language do not exist as a unitary phenomenon and, in consequence, cannot provide a window on language evolution. Returning now to the virtues of the Windows Approach, however: it encourages careful work on window phenomena. Specifically, looking at the evolution of language through relatively new windows demands that work on language evolution and work on the properties of window phenomena be done in tandem.

Which brings us to a first way in which diagram (6) offers an incomplete representation of the structure of windows on language evolution. It does not show that every window has to include an insightful theory of the window phenomenon referred to in box (a). The extent to which window inferences are grounded depends, after all, directly on how good such theories are. 


\section{Warrantedness}

Turning to warrantedness, it is a feature of the inferential step or steps represented by arrow (b) in schema (6). To see what this feature involves, observe that the question stated as (9) arises about the inferential steps allowed for by all windows on language evolution:

(9) Why is it proper to infer properties of some aspect of language evolution from properties of some window phenomenon?

This question asks for a warrant or licence for the inferential step or steps allowed by a window. With respect to the "degraded" language window, question (9) can be fleshed out as (10).

(10) What is it that warrants the inference of stages in the emergence of language from properties of "degraded" forms of language?

The warrant asked for in (10), obviously, cannot take the form of a bald stipulation to the effect that properties of "degraded" forms of language indicate that certain stages occurred in the emergence of language. Instead, this warrant will have to take the form of an empirical theory which gives a systematic account of how properties of "degraded" forms of language and (properties of) stages in the emergence of language are interlinked. The empirical theory needed will serve as a bridge by which to move inferentially from the domain of "degraded" language to the distinct domain of language evolution. ${ }^{10}$ In doing so, the required theory will offer a basis for answers to questions such as (10) and (11)(a) - (c).

(11) (a) Why does the fact - if fact it is - that Agent First and Focus Last occur in some "degraded" forms of language give these order principles their so-called evolutionarily primitive character, i.e. their language fossil status?

(b) Would every feature of every "degraded" form of language be evolutionarily primitive (and, thereby, reveal something about some stage in the emergence of language)?

(c) If the answer to (b) is "no", then what (kinds of) features of what "degraded" forms of language would be evolutionarily primitive or "language fossils"?

Questions such as (10) and (11)(a) - (c) - which are not considered by Jackendoff - point to a second way in which the "degraded" language window is less than well developed; it does not 
include any bridge theory which warrants the inferences in question. To construct a bridge theory that will be able to answer these questions, more empirical work on the properties of "degraded" forms of language will clearly have to be done.

We see here a second aspect of the heuristic potential of the Windows Approach to the study of language genesis. For it is the adoption of this approach that gives rise to questions such as (10) and (11)(a) - (c). Similar questions arise with respect to the inferential steps allowed for by other windows. As a matter of fact, all windows require bridge theories for warranting the inferences they allow. Unwarranted inferences are actually not inferences at all - they are stipulations. Or they represent speculations - to be underpinned by warrants at a later stage. This points to a second way in which the representation offered in diagram (6) of the structure of window inferences is incomplete. The diagram does not show that every window needs to include one or more bridge theories for warranting the conclusions it allows to be drawn. The extent to which these conclusions are warranted clearly depends on the merit of the bridge theories presupposed by them. Being typical instances of conclusions drawn in empirical inquiry, they cannot be necessarily true. In other words, to claim that a conclusion is warranted or licensed is not to claim that it is true.

\section{Pertinence}

Pertinence - or the lack of it - is a property of what is inferred by using a window on language evolution. That is, pertinence is a feature of the conclusions represented by box (c) in schema (6). In essence, a conclusion is pertinent to the extent that it is about what may be called "the right thing". The conclusions which are drawn by using Jackendoff's "degraded" language window are clearly about "the right thing": the evolution of language.

Which gives rise to the question: In what way could the conclusions of window inferences be about anything other than "the right thing"? How could such conclusions not be pertinent? There are two basic ways in which a conclusion of a window inference might not be pertinent: it might be about the evolution of an entity that is distinct from language or it might be about an aspect of language that is distinct from its evolution. Interestingly, recent literature provides various instances of window inferences whose conclusions have been challenged in regard to pertinence. Let us consider two of these instances in outline.

The first instance involves Tim Crow's $(2000,2002)$ "language" gene window. Looking at 
language evolution through this window, Crow concludes what is quoted in (12):

(12) " ... language evolved as a result of a [saltational - R.P.B.] genetic change that introduced a new principle of brain function." (Crow, 2002, p.3)

According to Crow, the genetic change in question occurred some 100,000 years ago and involved a critical change in a single gene for cerebral dominance on the $\mathrm{Y}$ chromosome of hominims. This change resulted in a shift in cerebral dominance which caused Homo sapiens to speciate suddenly with a lateralised brain. In Crow's view, this shift allowed language to evolve as a species-specific mate recognition system.

Crow's scenario for the evolution of language - and specifically his account of the crucial genetic change - has been criticized on various counts. ${ }^{11}$ One of these criticisms, articulated by Marian Annett $(1998,2000)$, is of direct interest to us here. She argues for the conclusion in (13) on the basis of evidence about children with hemiplegic cerebral palsy.

(13) (a) "The gene [that was involved in the change postulated by Crow - R.P.B.] is 'for' speech, not language." (Annett, 2000, pp.1-2)

(b) $\quad$... CD [i.e., cerebral dominance - R.P.B.] is not for high level language but for speech." (Annett, 2000, p.3)

In essence, what Annett argues is that Crow's inference quoted as (12) lacks the pertinence desired. That is, contrary to what Crow maintains, this inference is not about the evolution of the "right entity", namely language. It may be pertinent to the evolution of a distinct entity, namely speech, assuming that it is grounded, warranted and so on. Looking through the "language" gene window as constructed by Crow, one cannot draw directly pertinent inferences about the evolution of language - that is, if Annett and others have got it right.

A similar problem seems to come up in the context of Dean Falk's (2003) newly constructed motherese window. She proposes what she refers to as the "putting the baby down hypothesis". This hypothesis entails in Falk's own words what is stated in (14).

(14) "The central thesis regarding motherese is that bipedal mothers had to put their babies down next to them periodically in order to go about their business (of foraging R.P.B.] and that prosodic vocalizations would have replaced cradling arms as a means for keeping the little ones content." (Falk, 2003, p.20) 
These vocalisations - or so-called ancestral motherese - preceded in Falk's view what she refers to as "the first glimmerings of speech in early hominims" and as "the emergence of protospeech". What is problematic is that she goes on to refer to these vocalizations as "the evolutionary underpinnings that preceded the first glimmerings of language" and as "the prelinguistic vocal substrates for protolanguage". Significantly, in some contexts, Falk seems to draw a distinction between "protospeech" and "protolanguage" but in others she seems to consider them to be one and the same thing. This gives rise to doubts about the pertinence of the inferences she draws from data about motherese. Given that they are grounded and warranted, these inferences can be pertinent to the evolution of modern speech. But they cannot be directly pertinent to the evolution of modern language at the same time. It is possible, of course, that protospeech offers a window on the emergence of protolanguage. But using this further window would require one or more additional inferences which, in turn, would have to be grounded and warranted.

In terms of pertinence, Falk's motherese inferences and Crow's "language" gene inferences are problematic in the same way. What are generally taken to be two distinct linguistic entities language and speech - are squashed together in the case of both sets of inferences. This brings us to a point that applies to all window inferences: such inferences can be pertinent - that is, about the evolution of the "right entity", namely language - only if they are underpinned by a restrictive theory of what language is. Such a restrictive theory will provide a basis for discriminating in a non-arbitrary way between language and other linguistic entities that are distinct from it. $^{12}$

So, to return to diagram (6) and its representation of the structure of a window: that representation is incomplete - in a third way - in not making explicit provision for the restrictive theory of language which is needed if the conclusions in box (c) are to be pertinent in the sense of being about the "right entity". But note now that, even if they were about the "right entity", these conclusions would still fall short of being fully pertinent. To be fully pertinent, these conclusions have to be about the "right process", namely evolution, as well. So the requirement is that these conclusions have to be underpinned by a restrictive theory of evolution in addition to being underpinned by a restrictive theory of language. A restrictive theory of evolution will discriminate between processes of language evolution on the one hand (for example, those involved in the first appearance or in the subsequent development) 
and non-evolutionary processes of change on the other hand (for example, those involved in diachronic change or in dialect standardization).

The required theory of evolution has to dovetail with the required theory of language in a specific way. If the theory of language construes language as being a biological entity, then the theory of evolution has to be one of biological evolution. If, by contrast, the theory of language construes language as a social (or a cultural or some other type of) entity, then the latter theory has to be one of social (or cultural or some other type of) evolution. Moreover, the theory of evolution should be non-ad hoc. Thus, if it is, say, a theory of biological evolution, it should instantiate a general theory of (non-linguistic) biological evolution. The same goes, mutatis mutandis, for a theory of cultural (or some other type of) evolution.

Represented more fully, a window on language has the following structure:
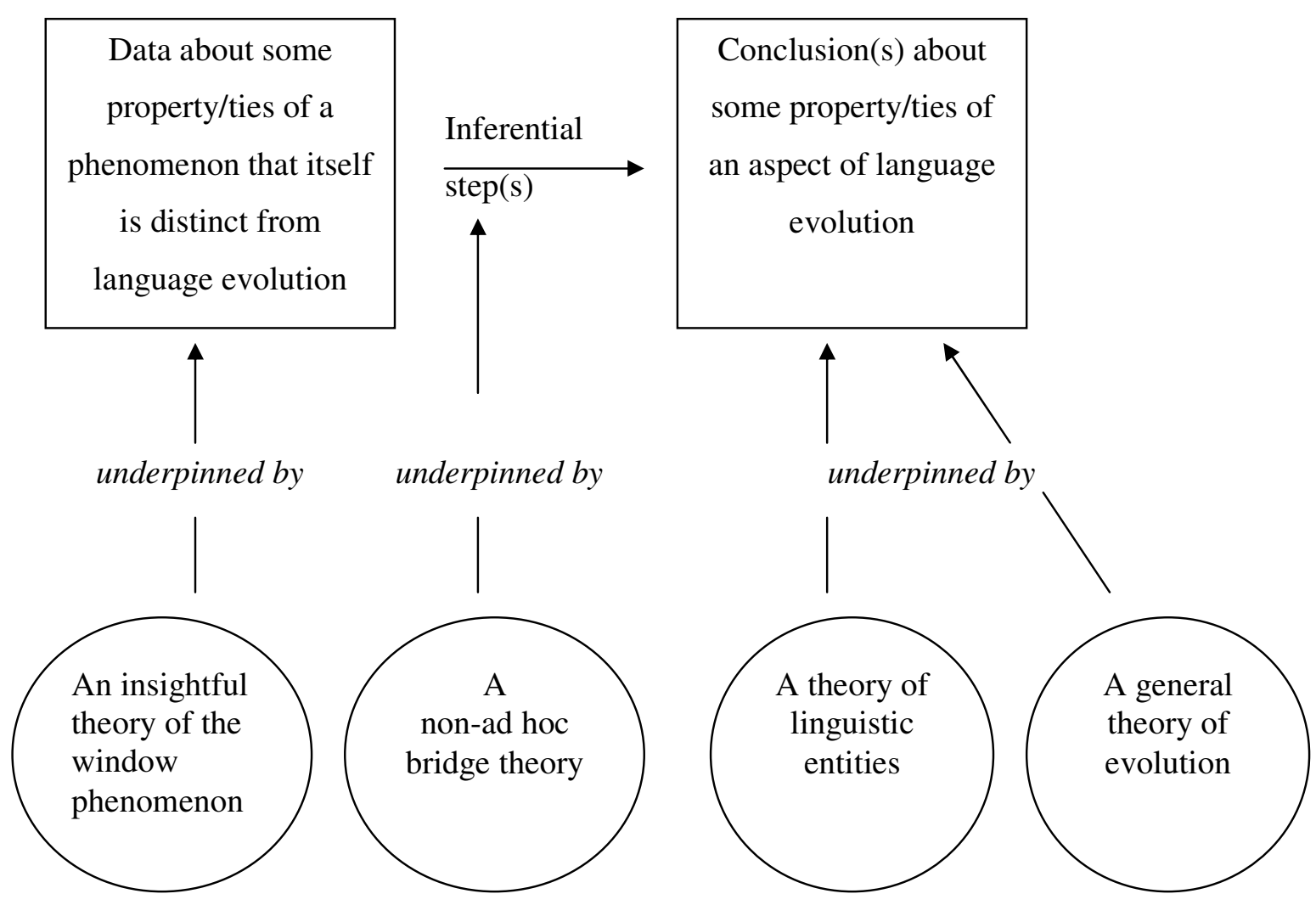

The characterizations offered by accounts of language evolution of such concepts as "the first language", "the first linguistic system", "ancestral language", "protolanguage", "true 
language" and "modern language", clearly, have to draw on a theory of linguistic entities and a theory of evolution as well - a point that is often overlooked.

\section{Coherence}

In the sections up to here, we have been looking at features of individual windows used in isolation of each other. In some accounts of language evolution, however, individual windows are strung together to form chains used for making inferences about language evolution on the basis of data about phenomena in domains that are far removed from that of language evolution. Series of inferences constructed with the aid of such window chains need to have the basic good-making property of coherence. To see what this property involves, let us now consider in outline the chain of windows used by Wendy Wilkins and Jenny Wakefield (1995, 1996) for inferring from data about (endocasts of) fossil skulls that the (neuroanatomical substrate of the) human language capacity emerged for the first time in Homo habilis some two million years ago.

In order to make the inferences in question, Wilkins and Wakefield use the following three windows: ${ }^{13}$

1. A fossil-skull window: from data about hollows, bumps and ridges in crania of fossil skulls, conclusions are drawn about grooves, furrows and the like that make up the sulcal pattern of Habilis brains.

2. A sulcal-pattern window: from conclusions about the sulcal pattern of Habilis brains, further conclusions are drawn about folds, lobes, areas - including the presence of Broca's area and the POT - in the anatomical organization of Habilis brains. ("POT" refers to a junction of three lobes of the brain: the parietal $(\mathrm{P})$, the occipital $(\mathrm{O})$ and the temporal (T). The POT is located in what is known as "Wernicke's area".)

3. An anatomical-organization window: from conclusions about the anatomical organization of Habilis brains, still further conclusions are drawn about the functional organization of Habilis brains and, in particular, about the presence of the human language capacity.

Wilkins and Wakefield use these three windows to make a series of inferences that may be schematically represented as below: 


\begin{tabular}{|c|}
\hline Data about \\
fossil \\
Habilis \\
skulls: \\
hollows, \\
bumps, \\
ridges etc. in \\
crania \\
\end{tabular}

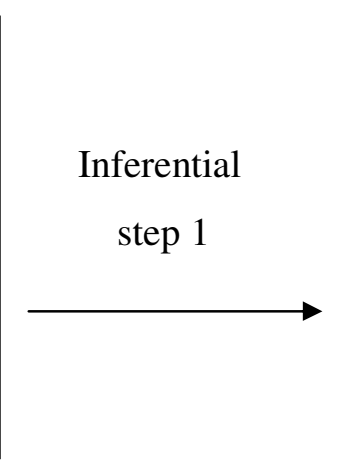

(b)

\begin{tabular}{|c|}
\hline Conclusions \\
about sulcal \\
pattern of \\
Habilis \\
brains: \\
grooves, \\
furrows etc. \\
on surface \\
\end{tabular}

(c)

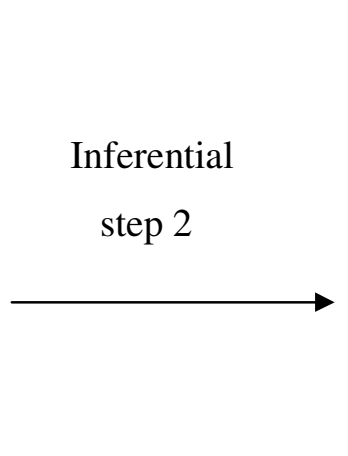

(d)

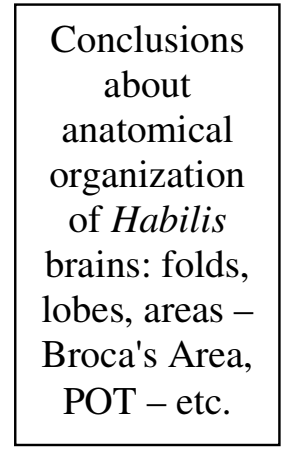

(e)

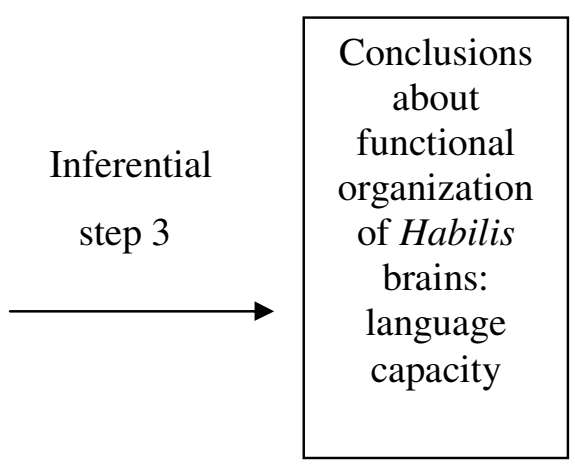

(f) 
To appraise this series of inferences, it has to be established for each of the three inferences whether it is properly grounded, warranted and pertinent. These inferences have, in fact, been criticized for lacking one or more of these good-making properties. It has been argued, for instance, that there are no facts about Habilis skulls in which the first inference can be grounded and that the localizationist neurolinguistic theory which is supposed to provide a warrant for the third inference is untenable. ${ }^{14}$

Appraising a series of inferences such as that reconstructed in (16) involves something more, however: it has to be established for each pair of consecutive inferences whether they cohere in a proper way. For the reconstruction in (16), this does indeed appear to be the case. Inferences (16)(b) and (d) cohere properly in that the conclusions of the first inference furnish the "basis" for the second one. And inferences (16)(d) and (f), likewise, cohere properly in that the conclusions of the second inference furnish the "basis" for the third one. That is, formally there is no gap between two consecutive inferences; the series of inferences is internally coherent. In passing, though, let us note here that there is something which such internal coherence of a series of inferences does not, and in fact cannot, do: it cannot compensate for a possible lack of groundedness, warrantedness or pertinence in any of its constituent inferences.

The series of inferences in (16) is about three (putative) windows on language evolution. In these (putative) windows, a noteworthy further property of the concept of "a window on language evolution" is illustrated: this concept can be used both in a restrictive sense and in a less restrictive sense. In terms of the restrictive sense, only the anatomical organization window "faces directly on to" language evolution: of the three windows in (16), only this one is a "direct" window on language evolution. The fossil-skull window is a "direct" window on the sulcal pattern of (Habilis) brains; the sulcal-pattern window, in turn, is a "direct" window on the anatomical organization of (Habilis) brains. These two windows are (putative) windows on language evolution in the less restrictive sense of forming constituents in a chain of windows of which only the last one is a (putative) window on language evolution in the restrictive sense. 


\section{A window with a view}

Let us consider then, for one last time, the question of the virtue of individual windows on language genesis and of the Windows Approach in general.

As for the Windows Approach to the study of language genesis, its virtue lies in the fact that it is heuristically powerful in two general ways. First: it represents a means of making potentially interesting and respectable inferences about what language genesis involved. Second: it generates questions which are highly specific and the pursuit of which is likely to lead to a deeper understanding of a wide range of "window" phenomena.

As for individual windows, what makes them virtuous, we have seen, are the groundedness, warrantedness and pertinence of the inferences about language genesis which they allow. A window that makes it possible to draw inferences which have these three features may aptly be called a "window with a view" - to give an unlicensed twist to the title of a famous EM Forster novel. 


\section{Notes}

1. In this connection see, for example, Klein (2001, pp. 85-87).

2. For these - and other - senses in which the concept of "language" has been used in recent work on language evolution, see Botha (2003, pp. 13-46).

3. For illustrations of these points, see Botha (2003, pp. 36-41).

4. See Botha (2003, pp. 64-65) for some of the consequences of not drawing the distinction between origin/emergence/first appearance and subsequent development in investigating language evolution.

5. See, for example, Kegl, Senghas and Coppola (1999) for the use of this expression for referring to some of the processes listed in (6).

6. For the use of the expression "indirect evidence", see, for example, Botha (2003, chap. 11).

7. I will refer below primarily to the former publication since, in ways that matter, it is more explicit than the latter. Jackendoff, incidentally, uses both the notion of a "step" and that of a "stage" in his discussion without clarifying the way in which they are interrelated.

8. Jackendoff refers to the "competence attained by Genie" and the "(linguistic) achievements of language-trained apes" as well, but it is not clear whether or not they are on his list.

9. For other problems with Jackendoff's notions of "language fossil" and "fossil evidence", see Botha (2003, pp. 200-201).

10. For the concept of a "bridge theory", see Botha (2003, pp. 147-150, 199-200).

11. For comments on the testability of this hypothesis, see Tyler-Smith (2002).

12. For the notion of a "restrictive theory of language", see Botha (2003, 43-45). Such a theory will draw a distinction between language on the one hand and linguistic entities such as forms of linguistic behaviour and linguistic skills on the other hand.

13. This represents a reconstruction of Wilkins and Wakefield's account. For specifics, see Botha (2003, pp. 142-147).

14. For specifics of these and other criticisms of the three inferences, see Botha 
(2003, pp. 142-150) and the literature cited there. 


\section{References}

Annett, M., 1998. Language, speech and cerebral dominance. CPC 17(6), 1118-1125.

Annett, M., 2000. No homo speciated on cerebral dominance. Psycholoquy:11, \#20 Language Sex Chromosomes (2).

Botha, R.P., 2003. Unravelling the Evolution of Language. Elsevier, Amsterdam etc.

Botha, R.P., 2004a. Windows on language evolution: What are they and Wherein lies their virtue? Stellenbosch Papers in Linguistics 35, pp. 1-21.

Botha, R.P., 2004b. Windows on language evolution: How are they constructed? Stellenbosch Papers in Linguistics 35, pp. 23-41.

Crow, T.J., 2000. Did Homo sapiens speciate on the X-chromosome? Psycholoquy:11, \#1 Language and sex chromosomes (1).

Crow, T.J., 2002. ProtocadherinXY: A candidate for cerebral asymmetry and language. In: Wray, A. (Ed.), The Transition to Language, Oxford University Press, Oxford, pp. 93-112.

Falk, D., 2003. Prelinguistic evolution in early homonims: Whence motherese? Behavioral and Brain Science, Text distributed by BBS for peer commentary.

Jackendoff, R., 1999. Possible stages in the evolution of the language capacity. Trends in Cognitive Science 3(7), pp. 272-279.

Jackendoff, R., 2002. Foundations of Language: Brain, Meaning, Grammar and Evolution. Oxford University Press, Oxford.

Kegl, J., Senghas, A., Coppola, M., 1999. Creation through contact: Sign language emergence and sign language change in Nicaragua. In: DeGraff, M. (Ed.), Language Creation and Language Change. Creolization, Diachrony, and Development, MIT Press, Cambridge, Massachusetts and London, pp. 179-237.

Klein W., 2001. Elementary forms of linguistic organization. In: Trabant, J., Ward, S. (Eds.), New Essays on the Origin of Language, Mouton de Gruyter, Berlin, pp. 81102.

Tyler-Smith, C., 2002. What can the Y chromosome tell us about the origin of modern humans? In: Crow, T.J. (Ed.), The Speciation of Modern Homo Sapiens. Oxford University Press, Oxford etc., pp. 217-229. 
Wilkins, W.K. Wakefield, J., 1995. Brain evolution and neurolinguistic preconditions. Behavioral and Brain Sciences 18, 161-182, 205-226.

Wilkins, W.K., Wakefield, J., 1996. Further issues in neurolinguistic preconditions. Behavioral and Brain Sciences 19, 793-798. 